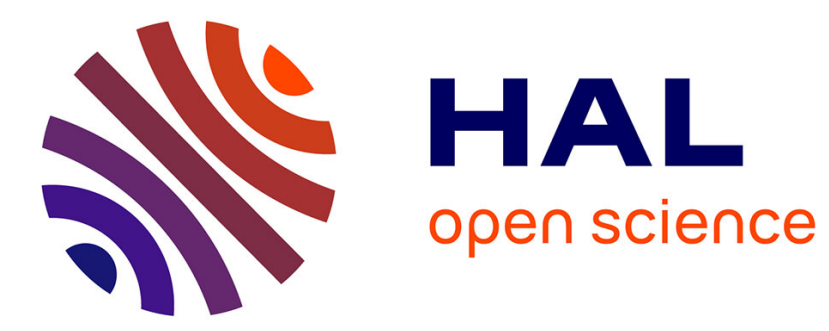

\title{
Polarimetric imaging beyond the speckle grain scale
}

Lucien Pouget, Julien Fade, Cyril Hamel, Mehdi Alouini

\section{To cite this version:}

Lucien Pouget, Julien Fade, Cyril Hamel, Mehdi Alouini. Polarimetric imaging beyond the speckle grain scale. Applied optics, 2012, 51 (30), pp.7345-7356. 10.1364/AO.51.007345 . hal-00761108

\section{HAL Id: hal-00761108 \\ https://hal.science/hal-00761108}

Submitted on 17 Dec 2014

HAL is a multi-disciplinary open access archive for the deposit and dissemination of scientific research documents, whether they are published or not. The documents may come from teaching and research institutions in France or abroad, or from public or private research centers.
L'archive ouverte pluridisciplinaire HAL, est destinée au dépôt et à la diffusion de documents scientifiques de niveau recherche, publiés ou non, émanant des établissements d'enseignement et de recherche français ou étrangers, des laboratoires publics ou privés. 


\title{
Polarimetric imaging beyond the speckle grain scale
}

\author{
Lucien Pouget, Julien Fade*, Cyril Hamel and Mehdi Alouini \\ Institut de Physique de Rennes, CNRS, Université de Rennes 1, Campus de Beaulieu, 35042 Rennes, France \\ * Corresponding author : julien.fade@univ-rennes1.fr \\ Compiled September 13, 2012
}

\begin{abstract}
We address an experimental Stokes imaging setup allowing one to explore the polarimetric properties of a speckle light field with spatial resolution well beyond the speckle grain scale. We detail how the various experimental difficulties inherent to such measurements can be overcome with a dedicated measurement protocol involving a careful speckle registration step. The setup and protocol are then validated on a metallic reference sample, and used to measure the state of polarization of light in each pixel of highly resolved speckle patterns (>2000 pixels per speckle grain) resulting from the scattering of an incident coherent beam on samples exhibiting different polarimetric properties. Evolution of the state of polarization with spatial averaging and across adjacent speckle grains is eventually addressed. (c) 2012 Optical Society of America

OCIS codes: (110.5405) Polarimetric imaging ; (260.5430) Polarization ; (110.6150) Speckle imaging ; (030.0030) Coherence and statistical optics ; (220.4830) Systems design
\end{abstract}

\section{Introduction}

Speckle is a ubiquitous phenomenon in all research fields studying the interaction between random media and propagating waves, whether electromagnetic [1-4] or acoustic [5]. Since speckle intensity patterns often represent the simplest and most accessible observables to gauge such interaction, this phenomenon has been thoroughly investigated for decades $[1,2,5]$ but is still widely studied in very active research fields of physics, such as wave localization [5], control of light through disordered media [6,7], optical non-linear effects in random media [8] or polarization singularities [9]. In the optical imaging domain, speckle has often been considered as a noise detrimental to image quality [10,11]. Nevertheless, speckle contrast imaging is known to be an efficient remote-sensing technique providing information on surface roughness properties [12-14] or fluid velocity [15] for instance. More recently, new applications involving speckle contrast images have been proposed to characterize polarization of light [16-19] or diffusion properties of materials [20,21].

Despite this intense research activity, an open debate still remains about how the polarization state distribution of a speckle pattern can be clearly linked with materials depolarization properties. Recently, this issue has occasioned a number of publications [20, 22-30], questioning for instance the definition and measurement of polarization correlation lengths in a spatial speckle pattern [22-24,28-30], or the partial "repolarization" of unpolarized coherent light backscattered by a depolarizing material [20]. In order to link depolarization properties of a sample with the scattered light polarization distribution, experimental studies have been carried out using a spatial multiple-scale analysis of the speckle polarization properties [23, 25-27]. In these experiments, light depolarization has been studied from a "macroscopic" point of view, by conducting a statistical analysis of a scalar pa- rameter (Degree Of Polarization (DOP)) [31] or (Orthogonal State Contrast (OSC)) [32], over a great number of coherence areas (speckle "grains"). These results tended to experimentally validate that the polarization state is deterministic (light is fully polarized) at the "local" scale of a single speckle grain, whereas global depolarization induced by interaction (reflection or transmission) with the sample results from spatial averaging on the detector over several coherence areas [23, 25-27].

However, the various experimental devices used in these references were not specifically dedicated to studying the polarization state of the speckle pattern at the local scale, i.e., beyond the speckle grain scale. To the best of our knowledge, an imaging setup capable of measuring the full Stokes vector (4 components) of light scattered by a diffusive sample below the speckle grain scale in the optical wave domain has not been clearly addressed in the literature. If such study could be carried out quite easily in the microwave range as suggested in [33], it turns out to be a mere challenge when the wavelength is only a few hundreds of nanometers, as already noticed in [9]. Indeed, Stokes measurements require polarizers and wave plates (at least one) to be inserted and rotated in front of the imaging detector, thus inevitably modifying the optical wavefront of the scattered light and hence the speckle pattern itself, as will be evidenced in the next section. This experimental difficulty is mostly often eluded in the literature, suggesting that conventional experimental schemes are not suitable to perform Stokes imaging at the speckle grain level. In this paper, we tackle this problem by proposing a rigorous experimental setup as well as the methodology allowing one to acquire Stokes images of a speckle pattern, where each grain covers several hundred or thousands of pixels on the detector surface. The paper is organized as follows: in Section 2, the experimental setup and measurement protocol proposed to achieve Stokes imaging be- 
yond the speckle grain scale are described and calibrated. Test samples are then described and characterized in Section 3. Lastly, we report in Section 4 experimental Stokes imaging of highly resolved speckle patterns obtained with this setup. The processing and analysis of these results are also addressed and commented in this section. Conclusions and future work directions are finally given in Section 5 .

\section{Experimental setup}

Analyzing the polarization properties of a speckle field beyond the speckle grain scale requires performing a measurement of the Stokes vector of light at any point of a highly resolved speckle pattern. Before detailing the experimental setup used, let us briefly recall the Stokes vector formalism and the principle of Stokes imaging.

\section{A. Stokes imaging principle}

Stokes formalism is commonly used to characterize the light state of polarization (SOP) [31]. According to this formalism, the polarization state of light is fully described by a four-component vector:

$$
\overrightarrow{\mathcal{S}}=\left(\begin{array}{c}
\mathcal{S}_{0}=I_{x}+I_{y} \\
\mathcal{S}_{1}=I_{x}-I_{y} \\
\mathcal{S}_{2}=I_{+45^{\circ}}-I_{-45^{\circ}} \\
\mathcal{S}_{3}=I_{R}-I_{L}
\end{array}\right)
$$

The four components of the Stokes vector $\mathcal{S}_{i}$ can thus be simply obtained from intensity measurements $\left(I_{x}, I_{y}, I_{+45^{\circ}}, I_{-45^{\circ}}, I_{R}, I_{L}\right)$ through six configurations of a polarization analyzer. In the context of polarimetric imaging considered in the paper, the polarization analyzer is used to record six intensity images on a detector matrix. From these six images, the SOP (i.e., the 4-component Stokes vector) in each pixel of the image is then determined. A global and scalar characterization of the SOP in each pixel is classically obtained by computing the degree of polarization (DOP) image, given by [31]

$$
D O P=\frac{\sqrt{\mathcal{S}_{1}^{2}+\mathcal{S}_{2}^{2}+\mathcal{S}_{3}^{2}}}{\mathcal{S}_{0}}=\sqrt{\mathrm{S}_{1}^{2}+\mathrm{S}_{2}^{2}+\mathrm{S}_{3}^{2}},
$$

or the orthogonal state contrast (OSC) given by

$$
O S C=\frac{\mathcal{S}_{1}}{\mathcal{S}_{0}}=\mathrm{S}_{1},
$$

where the $\mathrm{S}_{i}=\mathcal{S}_{i} / \mathcal{S}_{0}$ stand for the normalized Stokes components.

This method has been preferred to a Fourier analysis technique involving a rotating quarter-wave plate [31]. Indeed, this one implies recording multiple intensity snapshots through a moving plate, which is inconsistent with the extreme stability required for the experimental setup, as will be evidenced below. Another alternative would be using a liquid crystal variable retarder, but it would be necessary to check that no wavefront distortion is introduced when its configuration is changed during the measurement.

Indeed, to ensure validity of the polarimetric data recorded at the speckle grain scale, one needs to prevent any modification in the wavefront of the scattered light during the measurement procedure. Understandably, this last condition is not easily achieved since speckle is nothing but an interference pattern, and therefore any change in the optical path of the order of a fraction of wavelength will modify the speckle pattern. As will be detailed afterwards, unwanted wavefront distortions can be caused by air turbulence within the optical path, thermal expansions of the sample or mechanical vibrations, but the main difficulty is to keep the wavefront unchanged while switching between the six different configurations of the polarization analyzer. In the remainder of this section, we will address these technical issues in detail and propose an optimized experimental setup and measurement protocol allowing one to perform Stokes imaging beyond the speckle grain scale.

\section{B. Description of the experimental setup}

The experimental setup proposed is depicted in Figure 1. Stokes imaging of the speckle pattern is performed in a reflection geometry (quasi-monostatic configuration). Indeed, the polarimetric imaging system analyzes light scattered by the sample in a direction close to backscattering direction. Consequently, it has to be noted that in the remainder of this paper, the word backscattering will not refer to the strict sense of scattering in the incident beam direction. The setup comprises several building blocks which are described in detail below:

Illumination: In the experimental configuration chosen, the scattering sample is enlightened with a frequency doubled $\mathrm{Nd}: \mathrm{YVO}_{4}$ laser (Coherent Verdi) emitting a maximum output power of $2 \mathrm{~W}$ at $\lambda=532 \mathrm{~nm}$. Although the light emitted by the laser is linearly polarized, we use a Glan polarizer to ensure high contrast horizontal linear polarization of the illumination beam. The laser beam is then expanded and collimated with the association of a microscope objective $(\times 10,0.25 \mathrm{NA})$ and a convex lens $L_{0}\left(f_{0}^{\prime}=200 \mathrm{~mm}\right)$. A diaphragm is used to adjust the beam diameter and suppress unwanted diffusions or reflections on the edges of the collimation lens $\left(L_{0}\right)$. Finally, this beam forms a $2.5 \mathrm{~cm}$ diameter illumination spot on the scattering sample under test.

Imaging optics: A set of two converging lenses $L_{1}$ ( $\left.f_{1}^{\prime}=80 \mathrm{~mm}\right)$ and $L_{2}\left(f_{2}^{\prime}=40 \mathrm{~mm}\right)$ is used to image the surface of the sample on the detector plane. Such optical configuration makes it possible to position the polarization analyzer (PA) between the two lenses, in the intermediate image plane (IIP). The distance between the two lenses is $22.5 \mathrm{~cm}$ and the distance between $L_{2}$ and the intermediate image plane is set to $12 \mathrm{~cm}$. 
Though unusual in polarimetric imaging setups, positioning of the polarization analysis components in a conjugate plane of the sample and of the CCD is justified here since it helps minimizing wavefront deformations when the PA configuration is changed. Indeed, the beam has a minimum size in the intermediate image plane, forming an intermediate image of diameter $6.2 \mathrm{~mm}$ on the polarization analyzer. Provided the beam is well centered with the rotation axis of the PA, such configuration minimizes any spurious effect of planarity imperfections of the polarization analysis components (see paragraph Polarization analyzer below).

Imaging pinhole: To ensure a sufficient size of the coherence area on the detector, such that a speckle grain covers a few thousands of pixels, the aperture of the imaging system is shrunk by inserting a circular pinhole of diameter $\phi=200 \mu \mathrm{m}$ on the imaging lens $L_{2}$. The typical size of a speckle grain on the detector is then given by

$$
\delta=1.22 \lambda \frac{2 D}{\phi} \simeq 0.4 \mathrm{~mm}
$$

where $D=6 \mathrm{~cm}$ corresponds to the distance between imaging lens $L_{2}$ and the detector plane. In this configuration, each speckle grain of circular shape covers in the final image approximately 2300 square pixels of dimension $7.4 \mu \mathrm{m}$.

Imaging detector: Due to the tiny imaging aperture used in the experiment, the camera must exhibit good noise properties under low illumination levels. Consequently, we choose to record the images on a 12-bits monochromatic 659 x 494 pixels CCD camera (Basler scA640-70 fm). Moreover, to enhance the dynamic range, a dual exposure with two different acquisition times $\left(T_{2}=10 T_{1}\right)$ is used to extract data from the low intensity areas. Image processing is necessary to recombine the two snapshots, finally providing an intensity image with higher dynamics than the 4096 grayscale levels of the 12-bit camera (see pixels grayscale values of Fig. 9.a for instance).

Polarization analyzer (PA): Most conventional polarimetric imaging experiments use a single adjustable polarizer to measure $I_{x}, I_{y}, I_{+45^{\circ}}, I_{-45^{\circ}}$, and then add a quarter-wave plate to access the two images $I_{R}$ and $I_{L}$ associated with circular polarization of light. This simple and natural approach is not satisfactory in our experiment. Indeed, inserting an optical element between the sample and the CCD detector will necessarily modify the optical path of the backscattered light and modify the speckle pattern, thus inevitably spoiling the measurement as will be evidenced below. To maintain a constant optical path during the 6 intensity measurements, the polarization analyzer (PA) used consists of a nanoparticles linear film polarizer plate (2 $\mathrm{mm}$ thickness) and an achromatic quarter-wave plate (400-700 nm, $\lambda / 10$ wavefront distortion, $1 \mathrm{~mm}$ thick) closely packed together. These components are inserted in a single rotating optical mount with the axes of the polarizer and of the quarter-wave plate forming an angle of $45^{\circ}$. The relative positionning of the polarizer and quarter-wave plate, as well as the calibration of the PA axes were operated preliminary to any measurement, using the vertically polarized illumination laser beam. Calibration is an important step in the design of a polarimeter due to possible polarization artifacts introduced by the optical elements of the system [34].

To switch from a linear polarization analysis to a circular polarization analysis, the whole mount is turned around so that the analyzed light enters the polarizer first, or the quarter-wave plate first respectively. To this aim, the PA is set on a motorized rotating stage for precise and repeatable positioning. This configuration allows us to access the whole six intensity measurements needed to determine the full Stokes vector [31]. In addition, two angular degrees of freedom are added to the polarization analyzer mount to enable fine adjustment of the orientation of the polarization analysis plates with respect to the optical axis (One rotation about $\vec{e}_{x}$ and one about $\left.\vec{e}_{y}\right)$. The lens $L_{1}\left(f_{1}^{\prime}=80 \mathrm{~mm}\right)$ is also placed on a micrometric translation mount allowing fine positioning of the focal spot on the polarization analysis plates. These mechanical degrees of freedom associated to the PA are sketched in Figure 2. As will be evidenced in the next subsection, these additional degrees of freedom are indispensable to refine the relative positioning between the center of the PA and the focal spot in the intermediate image plane and thus minimize wavefront deformations between two successive acquisitions with different configurations of the PA.

Mechanical stability and housing: As will be detailed below, mechanical stability and air turbulence must be taken into account to ensure stability of the speckle pattern. To this end, the optical setup is compactly built on an optical table and breadboard. The whole setup (apart from laser) is inserted within a Plexiglas housing to protect it from air flows.

\section{Measurement protocol and speckle pattern registra- tion}

A crucial point to achieve Stokes imaging beyond the speckle grain scale is to ensure that the wavefront is not modified by the movement of the polarization analyzer during the complete Stokes measurement. In other words, when the configuration of the PA is switched to measure the different Stokes components, the speckle pattern must not change. As the PA is neither strictly plane nor placed in a plane strictly perpendicular to the optical axis, this condition is not a priori satisfied. This is evidenced in Figure 3 where a first Stokes intensity image $I_{x}$ is plotted (Fig. 3.a) and can be compared with a second intensity image $I_{+45^{\circ}}$ obtained after rotating 
the polarizer by $45^{\circ}$ angle (Fig. 3.b). As can be clearly seen, the speckle intensity pattern observed is obviously different. At this level, if wavefront distortions caused by $\mathrm{PA}$ rotation were to be ignored, such modification of the speckle intensity repartition could erroneously be interpreted as a signature of different SOPs across the speckle grains observed. Indeed, images of Figure 3 were recorded on a metallic slab sample. For such non depolarizing material, we have checked that the SOP after reflection is strictly identical to the illumination state. Consequently, the speckle intensity pattern must have different intensity levels but the same spatial repartition for each Stokes intensity measurement. Since this is clearly not the case in Fig. 3.b, one has to act on the fine PA orientation adjustments to recover the initial wavefront (or at least minimize the distortions). Acting very slightly and accurately on the three degrees of freedom of the polarization analyzer ( 2 rotations about $\vec{e}_{x}$ and $\vec{e}_{y}$ and the translation of the lens $L_{1}$ along $\vec{e}_{z}$ ), it is indeed possible to recover the initial speckle pattern reasonably well, as can be seen in Fig. 3.c. Such procedure will be referred to in the remainder of this article as speckle pattern registration.

Once evidenced the possibility of experimental speckle pattern registration, let us now detail the measurement protocol proposed to achieve Stokes imaging beyond the speckle grain scale. This protocol is based on the use of a metallic reference slab sample as follows:

- After a first intensity measurement, say $I_{x}$, is operated on a given sample, the metallic reference slab is inserted in front of the sample, in the vicinity of the sample surface. The speckle intensity pattern formed by the light backscattered on the metallic slab is temporarily stored as a reference.

- Then the PA is rotated to the next configuration to measure, say $I_{+45^{\circ}}$, thus inevitably causing wavefront distortions and hence modifying the current speckle intensity pattern.

- Registration of the speckle pattern is then carried out as described above, until the previously stored reference pattern is recovered. A coarse registration is first operated by trying to adjust the bright speckle grains, then a fine tuning is done by minimizing numerical criteria (spatial average of the whole difference image between the reference speckle pattern image and the current image (normalized in terms of average intensity) on the one hand, and root mean square of this difference image on the other hand). Once these two indicators are minimized, we can warrant that the initial wavefront has been recovered with minimum distortion.

- The metallic slab is then removed and the next image $\left(I_{+45^{\circ}}\right.$ in this example) can be eventually acquired on the sample under test.

To get a full characterization of the Stokes vector in each point of a highly resolved speckle pattern, the above protocol has to be followed between each Stokes intensity measurement to ensure that the same speckle pattern is analyzed all along the 6 acquisitions. This registration protocol can be quite long and tedious but is unavoidable. It must be noted that the speckle pattern might sometimes not be recovered if the mechanical or thermal drifts are not properly cancelled, as will be shown in the following subsection.

An illustration of the quality of speckle registration obtained during 4 successive Stokes measurements $I_{+45^{\circ}}, I_{-45^{\circ}}, I_{R}, I_{L}$, is given in Figure 4 . It can be observed that the registration procedure allows the speckle pattern to be recovered when the sample is non depolarizing (metallic reference) with various orientations of the PA.

\section{Air flow and thermal effects}

To minimize wavefront aberrations caused by air turbulence, the whole setup (apart from the laser) is packaged in a Plexiglas housing. However, modifications of the PA configuration and adjustment of the mount during speckle registration are done manually and it is thus necessary to keep a part of the housing open during the measurement process. The evolution during half an hour of the intensity of ten pixels arbitrarily chosen on a resolved speckle intensity pattern obtained on a metallic sample is plotted in Figure 5. During this experiment, the setup has been disturbed by some actions at various times indicated with vertical dotted lines in Figure 5. It can be seen that the speckle pattern is highly sensitive to these actions, indicating that handling of the PA mechanical mount must be operated with care. Moreover, we were able to measure a typical stabilization time of 50 seconds for the speckle field to stabilize to its initial pattern. This time was then considered as the typical time to wait between any intervention on the setup and the next measurement.

Besides, since imaging of the sample is operated through a small aperture (200 $\mu \mathrm{m}$ pinhole), it is thus necessary to illuminate the sample with a laser power of a few hundreds of milliWatts to warrant sufficient signalto-noise ratio on the detector. Taking into account the illumination spot diameter of $3.5 \mathrm{~cm}$, the illumination intensity is around $10 \mathrm{~mW} / \mathrm{cm}^{2}$. Despite this relatively moderate value, we found that thermal effects occur in the samples which undergo sufficient mechanical dilatation at a microscopic scale to alter the speckle interference intensity pattern acquired on the detector. It can indeed be seen in Fig. 5 that thermalization of the sample takes some time on a metallic slab (typically $>10$ minutes). This thermalization can be longer or even never reached on other samples, as illustrated in Figure 6 on a cardboard sheet sample. In this figure, the time evolution of the intensity of ten pixels in a resolved speckle intensity pattern is recorded during 30 minutes. As one can see, the speckle intensity pattern shows important variations during all the experiment, which are also imputed to bad mechanical stability of the sample. 
These experimental results indicate that the measurement of Stokes parameters on a highly resolved speckle intensity pattern requires a sufficient preliminary illumination time for the sample to thermalize (and the speckle pattern to stabilize). In addition, the samples have to show good thermal and mechanical stability to optimize the stability of the observed speckle pattern. For these reasons, the samples used in the following experiments have been deposited on a heavy marble block to help heat dissipation and to ensure mechanical stability. In these conditions, an acceptable stability of the speckle pattern can be obtained, which has been checked experimentally. It can indeed be observed in Figure 7 that after a 14 minutes (840 s) thermalization of a diffusive red paint sample deposited on a heavy marble block, the grayscale values of the ten pixels do not evolve by more than $5 \%$ for 6 minutes.

\section{Description of the experiment}

To illustrate the possibilities offered by the experimental setup and protocol described in the previous section, different experiments have been carried out by considering different samples with distinct scattering properties. Before displaying the experimental results obtained in Section 4, we describe the samples considered as well as their "macroscopic" (large spatial scale) depolarization properties given by the measurement of the Stokes parameters on non-resolved speckle patterns.

\section{A. Samples selection}

The samples considered have been selected for their distinct depolarization properties. First, a metallic slab has been used as a reference sample to check the validity of the method because of the well-known polarimetric behavior of such material. Next, two samples with distinct scattering characteristics have been designed for the purpose of the experiment by depositing a red and a green paint on a heavy marble block to warrant heat dissipation and mechanical stability as mentionned in the previous section.

On the one hand, in the case of the red paint sample, the green illuminating light $(\lambda=532 \mathrm{~nm})$ is rapidly absorbed at the sample surface. As a consequence, the small amount of backscattered light predominantly comes from surface reflection with a quasi-normal incidence angle and the incident SOP is thus maintained [35]. This is sketched in Fig. 8.a and this sample can thus be considered as non-depolarizing. On the other hand, in the case of the green paint sample, incident light is much less absorbed, enabling multiple scattering to occur in the bulk of the material, as illustrated in Fig. 8.b [35]. Such a volume scattering regime highly depolarizes the incident light, as will be checked in the next subsection. These two samples will make it possible to compare the effect of two different types of scattering on the local polarization below the speckle grain scale in the next section.

\section{B. Large scale polarimetric characterization of speckle patterns}

Large scale polarimetric characterization of the speckle fields backscattered by each sample has been carried out by measuring the values of the DOP and of the OSC in a measurement configuration where the speckle is not resolved, i.e., with each pixel of the camera averaging several speckle grains, as in standard coherent polarimetric imaging setups. For this purpose, we removed the pinhole in order to decrease speckle grains dimension below the pixel size. In this configuration, one can estimate how many speckle grains are averaged per pixel by determining the speckle contrast when backscattered light is fully polarized, which is given by [1]

$$
c=\frac{\langle I\rangle}{\sigma_{I}},
$$

where $\langle I\rangle=\left\langle\mathcal{S}_{0}\right\rangle$ is the mean intensity computed over the whole image and $\sigma_{I}=\sigma_{\mathcal{S}_{0}}$ is the standard deviation of the intensity. In this configuration, we measured a speckle contrast of 2.5, meaning that each pixel contains 2.5 speckle grains in average. We emphasize that in that case, as in standard coherent polarimetric imaging setups, the measurement of the Stokes parameters can be operated without the need of any speckle registration procedure. Indeed, uncontrolled wavefront distortions occurring during the measurement are wiped out by spatial averaging of several speckle grains in each pixel. Moreover, the Stokes vectors were estimated on homogeneous regions of 100 pixels (square regions of $10 \times 10$ pixels), thus increasing the effect of spatial averaging. Table 1 gives the results of the estimation on 420 subregions of 100 pixels of the normalized Stokes parameters $\mathrm{S}_{1}=O S C, \mathrm{~S}_{2}, \mathrm{~S}_{3}$ for each sample.

It can be noted that the values of the DOP and the OSC are similar for the metallic reference and the red paint sample. This confirms that these two materials share a similar non-depolarizing action and thus illustrates that light does not undergo multiple scattering in a surface scattering regime. For the green sample, the value of the DOP and of the OSC are much lower, which is in agreement with the expected depolarizing behavior of this sample due to volume scattering of light in the bulk of the material.

This large scale polarimetric characterization given in Table 1 will now be compared in the next section with polarimetric measurements beyond the speckle grain scale obtained with the setup and protocol presented in Section 2. More particularly, this preliminary characterization will allow us to analyze how "global" depolarization occurs from a spatial averaging of locally well-defined polarization states.

\section{Results and discussion}

\section{A. Stokes imaging beyond the speckle grain scale}

Let us now present the experimental results of Stokes imaging beyond the speckle grain scale obtained with the 
setup and protocol detailed in Section 2. These experimental acquisitions were conducted on the three samples described in the previous section. For each sample, 6 intensity images were recorded with a careful speckle registration step between each image acquisition, as described in Section 2.

Reference metallic slab: For the sake of concision, we only present the raw images obtained on the reference metallic slab sample in Figure 9. An example of raw image $I_{x}$ acquired with a horizontal linear polarization direction is given in Fig. 9.a. A region of interest (ROI) of $200 \times 150$ pixels is then selected in the initial image. In Fig. 9.b, the first Stokes image $\mathcal{S}_{0}$ is plotted and represents the total intensity of the speckle pattern as $\mathcal{S}_{0}=I_{x}+I_{y}$. One can notice that each speckle grain covers approximately a thousand pixels. Fig. 9.c shows the computed second normalized Stokes parameter $S_{1}$, which is equal to the OSC, as shown in Eq.(3). The third and fourth Stokes images are respectively plotted in Fig. 9.d and 9.e, and are finally used to determine the experimental DOP map, which is given in Fig. 9.f.

As expected, the values of the OSC and of the DOP are close to 1 across the ROI considered, while $\mathrm{S}_{2}$ and $\mathrm{S}_{3}$ maps are close to null values. This is particularly well verified in areas exhibiting a high intensity (bright speckle spots). Conversely, it decreases in the low intensity areas where DOP values exceeding 1 can be observed, thus revealing experimental biases in the measurement in spite of experimental care in the speckle registration procedure. As a consequence, these areas in the image should not be analyzed since the signal to noise ratio is too low to draw any conclusion.

Other samples: The experimental results obtained on the two other samples considered are given in Figure 10 which provides the total intensity image (Stokes parameter $\mathcal{S}_{0}$ ) as well as the computed OSC and DOP maps on a $200 \times 150$ pixels ROI. It can be checked in Fig. 10.a, Fig. 10.c, and Fig. 10.e that the red sample exhibits a behavior strongly similar to the metallic sample with DOP and OSC values close to 1 in high intensity areas. This non depolarizing behavior is in agreement with the expected surface scattering regime, and with the large-scale polarimetric measurements presented in Subsection B. Moreover, this figure thus confirms experimentally that incident polarization is maintained during interaction with the material in a surface scattering regime.

In the case of the green paint sample, it can first be observed in Fig. 10.d that the SOP of light is well-defined within each coherence area (speckle grain) since the value of the DOP remains close to 1 in each pixel in a bright speckle spot. This is in agreement with theoretical predictions and previous experimental hints obtained from multiple-scale analyses of non resolved speckle intensity patterns [23,25-27]. By contrast, unlike the red paint and metallic samples, it can be seen in Fig. 10.f that for the green paint sample, the initial polarization is not neces- sarily maintained in all the coherence areas since OSC values significantly lower than unity can be observed in areas corresponding to bright speckle spots with DOP values close to 1 .

In the next subsection, these experimental results are analyzed with more details through the mapping of the spatial repartition of the SOPs on Poincaré's sphere.

\section{B. Analysis of polarization states spatial repartition}

To complement the study of the spatial repartition of local SOPs within the speckle pattern, it is possible to map the normalized Stokes components of each pixel on a Poincaré's sphere as shown in Figure 11. In Fig. 11.a, the repartition of the pixels SOP obtained on the reference metallic slab in the whole ROI defined in Fig. 9.a is plotted on Poincaré's sphere, whereas Fig. 11.b and 11.c represent the pixels SOP in sub-ROIs 1 and 2 defined in Fig. 9.b. The colors associated with each point on the sphere denote the total intensity (parameter $\mathcal{S}_{0}$ ) of the corresponding pixel, in agreement with pixel colors of Fig. 9.b. As a consequence, a larger degree of confidence can be granted to the red points, corresponding to the pixels of the bright speckle grains but the SOPs for the darkest pixels plotted in blue must be interpreted warily due to significant experimental noise. In addition, the red circle corresponds to the mean value of the Stokes vector estimated in the whole region of interest whereas the black circle shows the value of the Stokes vector determined from the preliminary large scale polarimetric characterization presented in Subsection B and given in Table 1. From Figures 11.b and 11.c, it can be observed that the two speckle grains share the same SOP, corresponding almost perfectly to the incident illumination polarimetric state and to the "global" polarimetric state observed after spatial averaging (Fig. 11.a). This confirms the non-depolarizing behavior of this metallic reference sample as every single speckle is in the same polarization state. We highlight again that the important dispersion of the SOPs plotted in blue is due to important experimental noise in darkest regions of the speckle patterns.

A similar behavior is confirmed for the red paint sample by analyzing the polarimetric repartition over Poincaré's sphere given in Fig. 11.d for the whole ROI and in Fig. 11.e and 11.f for sub-ROIs 1 and 2 defined in Fig. 10.a. As is the case for the metallic sample, the two speckle grains selected in sub-ROIS 1 and 2 share the same SOP, matching with the illumination polarization, and the "global" polarimetric state measured after spatial averaging.

In the case of the green paint sample, the analysis of the spatial mapping of SOPs over Poincaré's sphere is more interesting. It can first be checked that after averaging over the whole ROI, the mean polarimetric state represented by the red circle in Fig. 11.g is in agreement with the "global" polarization measured in Subsection $\mathrm{B}$ after spatial averaging (black circle). Averaging over the ROI indeed yields a globally depolarized sate 
( $\mathrm{DOP}=0.44$ in average from Table 1$)$, corresponding to a point lying inside Poincaré's sphere. However, at a single speckle grain scale, the SOP is well defined. Indeed, as can be seen in Figures 11.h and Fig. 11.i which correspond respectively to the SOP of the pixels in sub-ROIs 1 and 2 defined in Fig. 10.b, the DOP is close to 1 and the polarization state lies on the surface of Poincaré's sphere. In addition, contrarily to the previous samples, one can see that the two speckle grains considered exhibit different SOPs, which do not exactly resemble the illumination polarization state. Such behavior is a consequence of multiple scattering occurring in the bulk of the sample in the case of the green paint sample, as explained in Section A.

These results thus represent a clear experimental evidence in the optical domain and beyond the speckle grain scale that light is fully polarized at a local scale, whatever the scattering regime considered (surface or bulk). As suggested by previous results obtained from a statistical analyses of polarization states on non-resolved speckle patterns [23, 27], global depolarization properties stem from a spatial averaging of local SOPs over several coherence areas. Using the experimental results gathered, we analyze this property in the next subsection.

\section{Multiple-scale analysis of the degree of polarization}

A multiple-scale analysis of the degree of polarization has been conducted on the highly resolved Stokes images measured. The results are summarized in Table 2 which contains the values of the DOP and the OSC averaged over the whole ROI after local binning with different binning pitches. For instance, a binning pitch of 30 means that 33 blocks of $30 \times 30$ pixels have been averaged in a ROI of $200 \times 150$ pixels. As the binning pitch is increased, it can be seen that DOP and OSC values tend to the values measured in Section B (see Table 1), which is in agreement with previous works [23,27]. This result, which is better evidenced in the case of the green paint sample, thus tends to confirm that the global depolarizing behavior emerges from a spatial averaging of varying polarization states, well-defined at a local scale.

\section{SOP mapping across speckle pattern - Observation of an adiabatic polarization transition}

To illustrate the potentialities offered by the setup and protocol proposed in this paper, let us lastly present how the spatial evolution of the SOP can be mapped along a geometrical path across the speckle pattern studied. Let us for instance consider the two contiguous speckle grains corresponding to the sub-ROIs defined for the green paint sample in Fig. 10.b. This case is indeed interesting since it has been checked in the previous section and in Fig. 11 that these two speckle grains exhibit different SOPs but a DOP close to unity. With the setup proposed, it is possible to study how this polarization evolves from one speckle grain to the other. The data plotted in Fig. 12.a on Poincaré's sphere corresponds to the evolution of the SOP along the black dotted line drawn in Fig. 10.b. On the sphere, the dark points correspond to the pixels located at the beginning of the line and the bright points to those located at the tip of the black dashed arrow of Fig. 10.b. The OSC and DOP measured along this line are also plotted respectively with dashed and plain line in Fig. 12.b. From these results, we can see that the spatial SOP transition between two speckle grains is continuous, which is consistent with previous works $[9,36]$. In the situation considered, the incident illumination SOP which is almost maintained in the first speckle grain then rapidly evolves towards a distinct polarization state which does not resemble the initial laser polarization. Nevertheless, this evolution is shown to be adiabatic so that the SOP follows a continuous trajectory along the surface of Poincaré's sphere.

\section{Conclusion}

In this article, we addressed the experimental issue of coherent active Stokes imaging beyond the speckle grain scale, i.e., on a highly resolved speckle pattern with each speckle grain covering several hundreds or thousands of pixels. The numerous experimental difficulties encountered to achieve such a measurement have been identified and solved to design the first realization, to the best of our knowledge, of a polarimetric imaging setup dedicated to such measurement. In addition, we proposed an experimental protocol able to warrant a constant wavefront during the entire measurement process. We demonstrated that the setup and the speckle registration protocol proposed allow the measurement of the full Stokes vector to be achieved in each pixel of a highly resolved speckle pattern. After a validation of the technique on a metallic reference sample exhibiting a wellknown non-depolarizing behavior, experimental Stokes imaging beyond the speckle grain scale has been demonstrated on two scattering samples exhibiting different scattering regimes (surface and bulk). The influence of these distinct scattering regimes on the local polarization state of light has been studied and commented, and the conclusions obtained are in agreement with previous experimental works using a statistical analysis on moderately resolved speckle pattern. Lastly, these experiments allowed us to experimentally demonstrate in the optical domain that the polarization transition between two contiguous speckles follows a continuous and adiabatic evolution.

There are various perspectives to this work among which we can cite a more thorough study of SOP transitions between adjacent speckle grains and the analysis of polarimetric correlation lengths in regard to speckle intensity coherence areas. The investigation of the influence of the source wavelength and coherence on polarization beyond the speckle grain scale is currently under progress. Besides, the analysis of specific samples exhibiting non standard scattering properties is also an interesting perspective. From an applicative point of view, we are quite convinced that exploring the polarization 
properties at a microscopic scale could lead to a more accurate understanding of the physics happening in optical systems or techniques using the speckle $[6,7,16-19,21]$ and thus might help in optimizing these systems. All these perspectives would benefit from the development of more robust experimental designs, by automating the speckle registration optimization stage for instance (programmable actuators, liquid-crystal polarimetric devices, ...) or even using a four-arm division of amplitude system with appropriate phase plates/polarizers and calibration in order to perform simultaneous imaging of the four Stokes components. 


\section{References}

1. J. W. Goodman, Speckle phenomena: Theory and Applications (Roberts \& Company Publishers, Greenwood village, 2007).

2. M. I. Skolnik, Introduction to Radar Systems : Third Edition (Mc Graw-Hill, 2001).

3. J. K. Jao, "Amplitude distribution of composite terrain radar clutter and the K-distribution," IEEE Trans. on Antennas and Propagation AP-32, 1049-1062 (1984).

4. N. Garcia and A. Z. Genack, "Crossover to strong intensity correlation for microwave radiation in random media," Phys. Rev. Lett. 63, 1678-1681 (1989).

5. P. Sheng, Introduction to wave scattering, localization and mesoscopic phenomena, 2nd ed. (Springer, 2006).

6. I. Freund, "Time-reversal symmetry and image reconstruction through multiple-scattering media," J. Opt. Soc. Am. A 9, 456-463 (1992).

7. S. M. Popoff, G. Lerosey, R. Carminati, M. Fink, A. C. Boccara, and S. Gigan, "Measuring the transmission matrix in optics: An approach to the study and control of light propagation in disordered media," Phys. Rev. Lett. 104, 100601 (2010).

8. W. H. Peeters, J. J. D. Moerman, and M. P. van Exter, "Observation of two-photon speckle patterns," Phys. Rev. Lett. 104, 173601 (2010).

9. O. V. Angelsky, I. I. Mokhun, A. I. Mokhun, and M. S. Soskin, "Interferometric methods in diagnostics of polarization singularities," Phys. Rev. E 65, 036602 (2002).

10. M. Alouini, F. Goudail, A. Grisard, J. Bourderionnet, D. Dolfi, A. Bénière, I. Baarstad, T. Løke, P. Kaspersen, $\mathrm{X}$. Normandin, and G. Berginc, "Near-infrared active polarimetric and multispectral laboratory demonstrator for target detection," Appl. Opt. 48, 1610-1618 (2009).

11. A. Bénière, M. Alouini, F. Goudail, and D. Dolfi, "Design and experimental validation of a snapshot polarization contrast imager," Appl. Opt. 48, 5764-5773 (2009).

12. D. Léger, E. Mathieu, and J. C. Perrin, "Optical surface roughness determination using speckle correlation technique," Appl. Opt. 14, 872-877 (1975).

13. M. Lehman, J. A. Pomarico, and R. D. Torroba, "Digital speckle pattern interferometry applied to a surface roughness study," Optical Engineering 34, 1148-1152 (1995).

14. R. A. Sprague, "Surface roughness measurement using white light speckle," Appl. Opt. 11, 2811-2817 (1972).

15. N. Andrés, M. P. Arroyo, H. Hinrichs, and M. Quintanilla, "Digital speckle-pattern interferometry as a fullfield fluid-velocimetry technique," Optics Letters 24, 575-577 (1999).

16. I. Freund, "Stokes-vector reconstruction," Opt. Lett. 15, 1425-1427 (1990)

17. M. Roche, J. Fade, and P. Réfrégier, "Parametric estimation of the square degree of polarization from two intensity images degraded by fully developed speckle noise," J. Opt. Soc. Am. A 24, 2719-2727 (2007).

18. P. Réfrégier, J. Fade, and M. Roche, "Estimation precision of the degree of polarization from a single speckle intensity image," Opt. Lett. 32, 739-741 (2007).

19. J. Fade, M. Roche, and M. Alouini, "Computational polarization imaging from a single speckle image," Optics Letters 88 (2012)
20. J. Sorrentini, M. Zerrad, G. Soriano, and C. Amra, "Enpolarization of light by scattering media," Opt. Express 19, 21313-21320 (2011).

21. N. Curry, P. Bondareff, M. Leclercq, N. F. van Hulst, R. Sapienza, S. Gigan, and S. Grésillon, "Direct determination of diffusion properties of random media from speckle contrast," Opt. Lett. 36, 3332-3334 (2011).

22. T. Setälä, A. Shevchenko, M. Kaivola, and A. T. Friberg, "Polarization time and length for random optical beams," Phys. Rev. A 78, 033817 (2008).

23. J. Broky and A. Dogariu, "Complex degree of mutual polarization in randomly scattered fields," Opt. Express 18, 20105-20113 (2010).

24. J. Broky and A. Dogariu, "Correlations of polarization in random electro-magnetic fields," Opt. Express 19 15711-15719 (2011).

25. J. Li, G. Yao, and L. V. Wang, "Degree of polarization in laser speckles from turbid media: implication in tissue optics," Journal of Biomedical Optics 7, 307-312 (2002).

26. C. Amra, M. Zerrad, L. Siozade, G. Georges, and C. Deumié, "Partial polarization of light induced by random defects at surfaces or bulks," Opt. Express 16, 10372-10383 (2008).

27. M. Zerrad, J. Sorrentini, G. Soriano, and C. Amra, "Gradual loss of polarization in light scattered from rough surfaces: Electromagnetic prediction," Opt. Express 18, 15832-15843 (2010).

28. J. Ellis and A. Dogariu, "Complex degree of mutual polarization," Opt. Lett. 29, 536-538 (2004).

29. O. Korotkova and E. Wolf, "Generalized Stokes parameters of random electromagnetic beams," Opt. Lett. 30 , 198-200 (2005).

30. J. Tervo, T. Setälä, A. Roueff, P. Réfrégier, and A. T. Friberg, "Two-point stokes parameters: interpretation and properties," Opt. Lett. 34, 3074-3076 (2009).

31. E. Collett, Polarized light: fundamentals and applications (Dekker, New-York, 1993).

32. S. Breugnot and P. Clémenceau, "Modeling and performances of a polarization active imager at $\lambda=806 \mathrm{~nm}$," Opt. Eng. 39, 2681-2688 (2000).

33. S. Zhang, Y. D. Lockerman, J. Park, and A. Z. Genack, "Interplay between generic and mesoscopic speckle statistics in transmission through random media," Journal of Optics A: Pure and Applied Optics 11, 094018 (2009).

34. E. Compain, S. Poirier, and B. Drevillon, "General and self-consistent method for the calibration of polarization modulators, polarimeters, and mueller-matrix ellipsometers," Appl. Opt. 38, 3490-3502 (1999).

35. M. Alouini, F. Goudail, N. Roux, L. Le Hors, P. Hartemann, S. Breugnot, and D. Dolfi, "Active spectro-polarimetric imaging: signature modeling, imaging demonstrator and target detection," The European Physical Journal - Applied Physics 42, 129-139 (2008).

36. M. S. Soskin, V. Denisenko, and I. Freund, "Optical polarization singularities and elliptic stationary points," Opt. Lett. 28, 1475-1477 (2003). 


\section{FIGURE CAPTIONS}

Figure 1: Experimental setup (See text for details).

Figure 2: Schematic description of the PA in a linear analyzer configuration. The PA mechanical mount provides two angular degrees of freedom (rotation $\theta$ about axis $\vec{e}_{X}$ and rotation $\psi$ about axis $\vec{e}_{Y}$ ) used during the speckle registration procedure.

Figure 2: Reference speckle intensity pattern (a) acquired on a metallic slab. Wavefront distortions caused by rotation of the PA modify the speckle intensity pattern (b) which can however be fairly recovered (c) after speckle registration.

Figure 3: Contour plots: speckle intensity patterns observed after speckle registration with 4 orientations of the PA on a metallic reference slab. Grayscale background image: total intensity image $\mathcal{S}_{0}$.

Figure 4: Time evolution of the intensity of ten pixels in a speckle intensity pattern with perturbations when the sample is a metallic slab. 1: cover is opened and air is blown inside. $2: 360^{\circ}$ rotation about $\vec{e}_{z}$ of the PA. 3 : $360^{\circ}$ rotation about $\vec{e}_{y}$ of the $\mathrm{PA}$.

Figure 5: Time evolution (30 mins) of the intensity of ten pixels when the sample is a cardboard sheet sample.

Figure 6: Time evolution (20 mins) of the intensity of ten pixels when the sample is a red paint deposited on a heavy marble block.

Figure 7: Schematic illustration of (a) surface and (b) volume scattering regimes obtained by scattering of a green illumination on red and green samples respectively. The white arrows symbolize the electric field polarization direction.

Figure 8: Stokes imaging of a speckle intensity pattern obtained on the metallic reference slab. (a) : example of raw image acquisition $\left(I_{x}\right)$ and selection of the ROI. From the 6 acquisitions, the 4 Stokes images are deter- mined: (b) First Stokes image $\mathcal{S}_{0}$ (total intensity). Two sub-ROIs are defined to compare the SOP in distinct speckle grains ; (c) Second normalized Stokes image $S_{1}$; (d) Third normalized Stokes image $\mathrm{S}_{2}$; (e) Fourth normalized Stokes image $\mathrm{S}_{3}$; (f) Degree of polarization. Total intensity repartition is indicated in contour plots in (c),(d),(e) and (f) thumbnails.

Figure 9: Stokes imaging beyond the speckle grain scale of a speckle intensity pattern obtained on a red paint sample [(a), (c) and (e)] and a green paint sample [(b), (d) and (f)]. (a), (b): First Stokes image $\mathcal{S}_{0}$ (total intensity). Two sub-ROIs are defined to compare the SOP in distinct speckle grains ; (c), (d): Map of the computed DOP ; (e), (f): Map of the computed OSC. Total intensity repartition is indicated in contour plots in (c), (d), (e) and (f) thumbnails.

Figure 10: Repartition of the pixels SOP on Poincaré's sphere for a metallic sample (first line), a red paint sample (second line) and a green paint sample (third line). First column: SOP in the whole ROI; Second column: SOP in sub-ROI 1 ; Third column: SOP in sub-ROI 2. Sub-ROIs are respectively defined in Fig. 9.b, Fig. 10.a and Fig. 10.b.

Figure 11: Observation of the adiabatic polarization state transition along the geometrical path defined in Fig. 10.b between two adjacent speckle grains on the green paint sample exhibiting bulk scattering regime ; (a) Evolution of the SOP on the Poincaré's sphere; (b) Evolution of the OSC (dotted line) and DOP (plain line). 


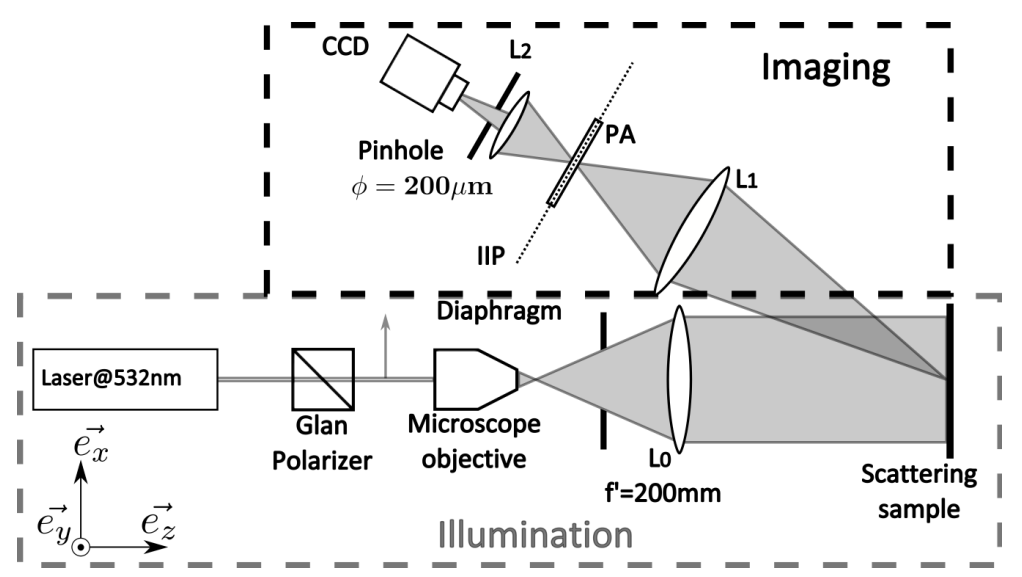

Fig. 1. Experimental setup (See text for details). 


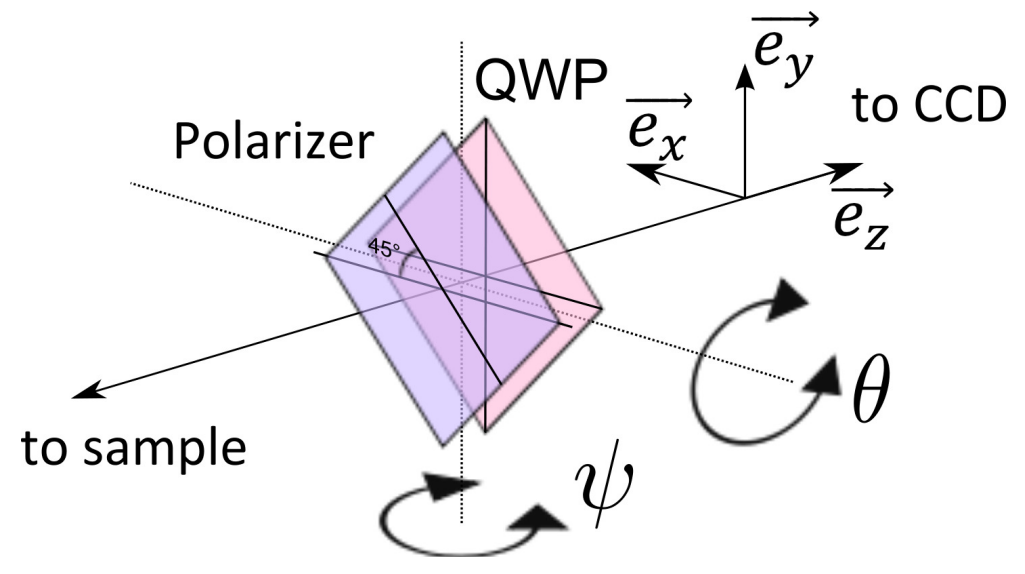

Fig. 2. Schematic description of the PA in a linear analyzer configuration. The PA mechanical mount provides two angular degrees of freedom (rotation $\theta$ about axis $\vec{e}_{X}$ and rotation $\psi$ about axis $\vec{e}_{Y}$ ) used during the speckle registration procedure. 


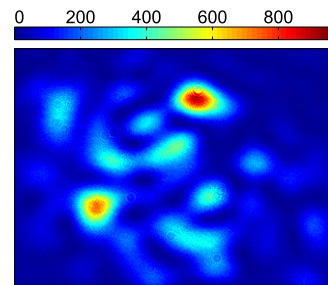

(a)

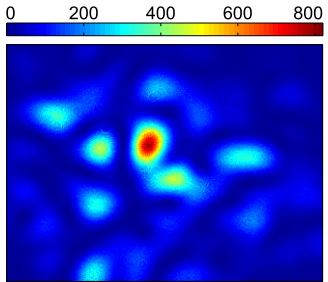

(b)

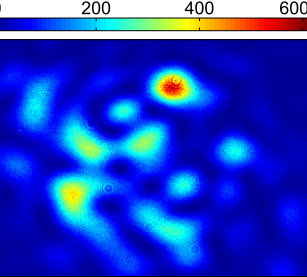

(c)

Fig. 3. Reference speckle intensity pattern (a) acquired on a metallic slab. Wavefront distortions caused by rotation of the PA modify the speckle intensity pattern (b) which can however be fairly recovered (c) after speckle registration. 


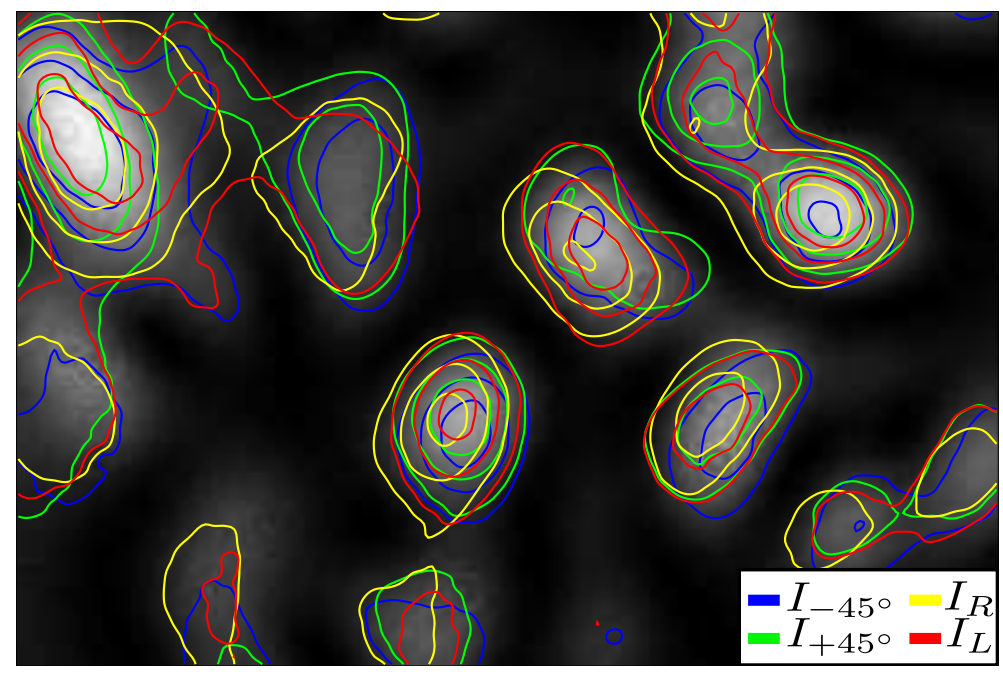

Fig. 4. Contour plots: speckle intensity patterns observed after speckle registration with 4 orientations of the PA on a metallic reference slab. Grayscale background image: total intensity image $\mathcal{S}_{0}$. 


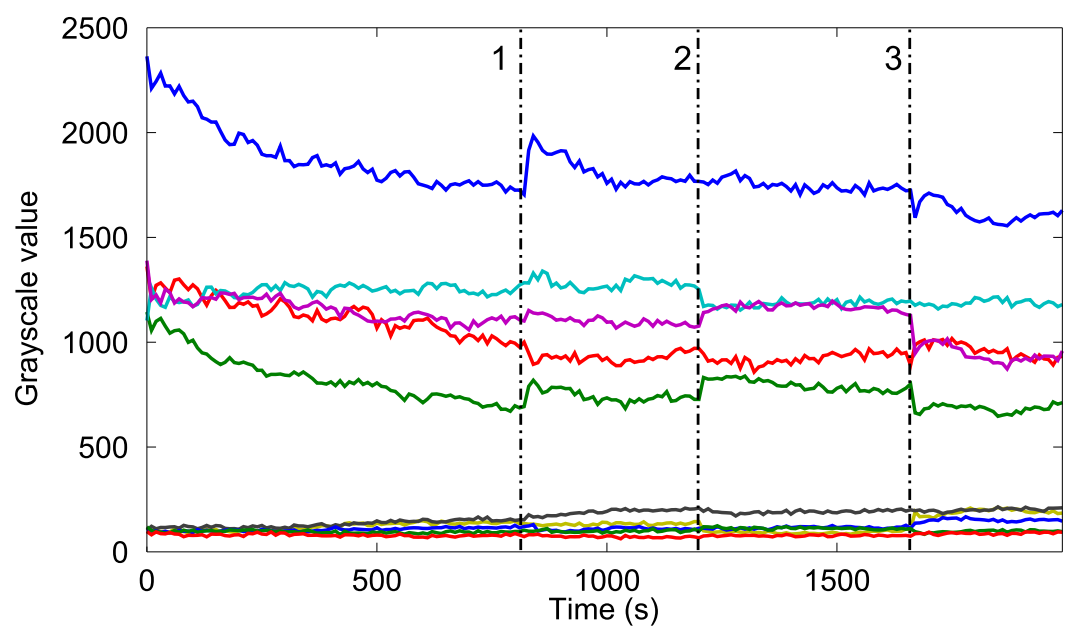

Fig. 5. Time evolution of the intensity of ten pixels in a speckle intensity pattern with perturbations when the sample is a metallic slab. 1: cover is opened and air is blown inside. $2: 360^{\circ}$ rotation about $\vec{e}_{z}$ of the PA. 3: $360^{\circ}$ rotation about $\vec{e}_{y}$ of the $\mathrm{PA}$. 


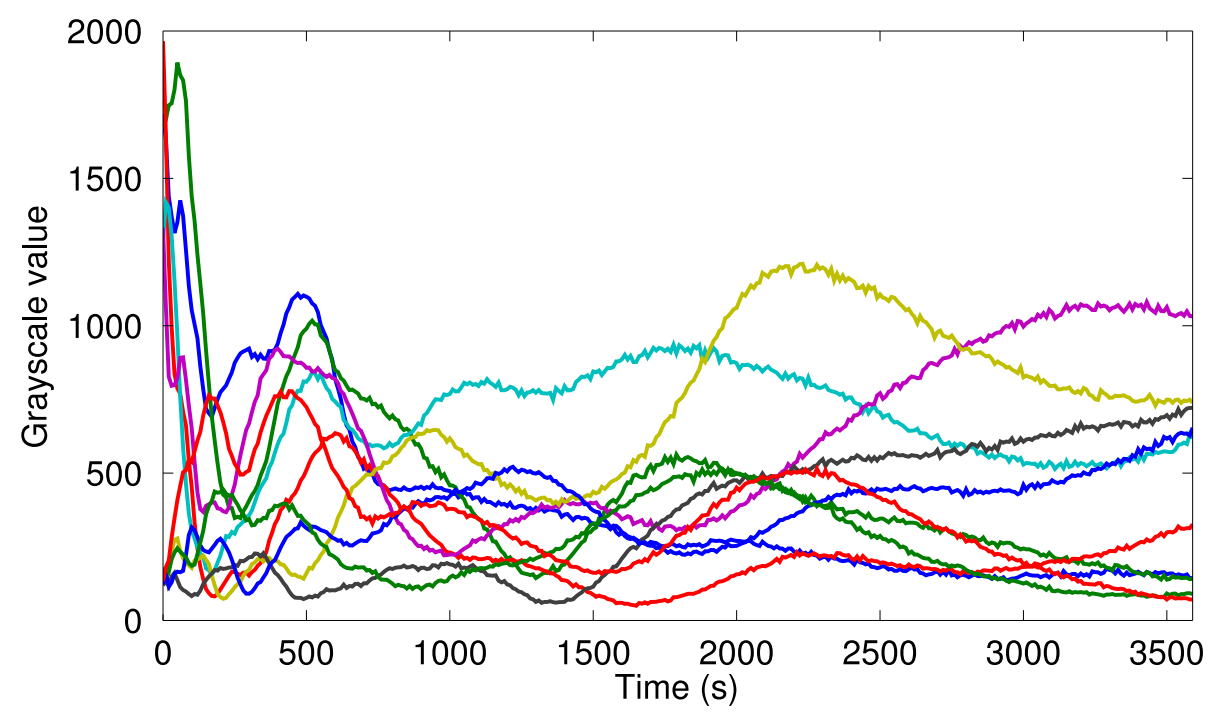

Fig. 6. Time evolution (30 mins) of the intensity of ten pixels when the sample is a cardboard sheet sample. 


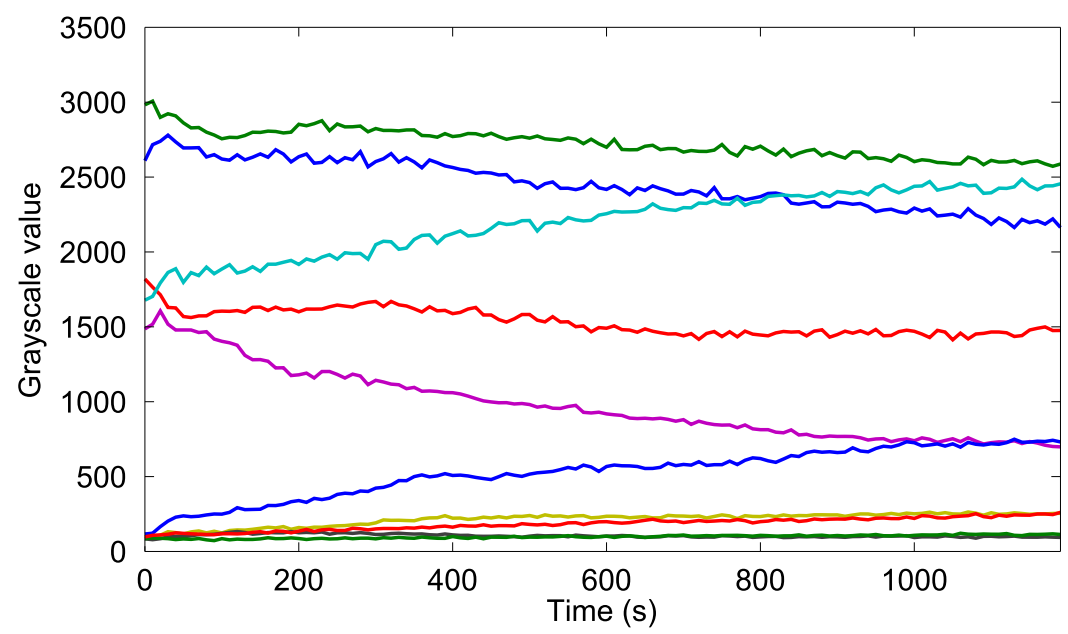

Fig. 7. Time evolution (20 mins) of the intensity of ten pixels when the sample is a red paint deposited on a heavy marble block. 


$$
\forall V
$$



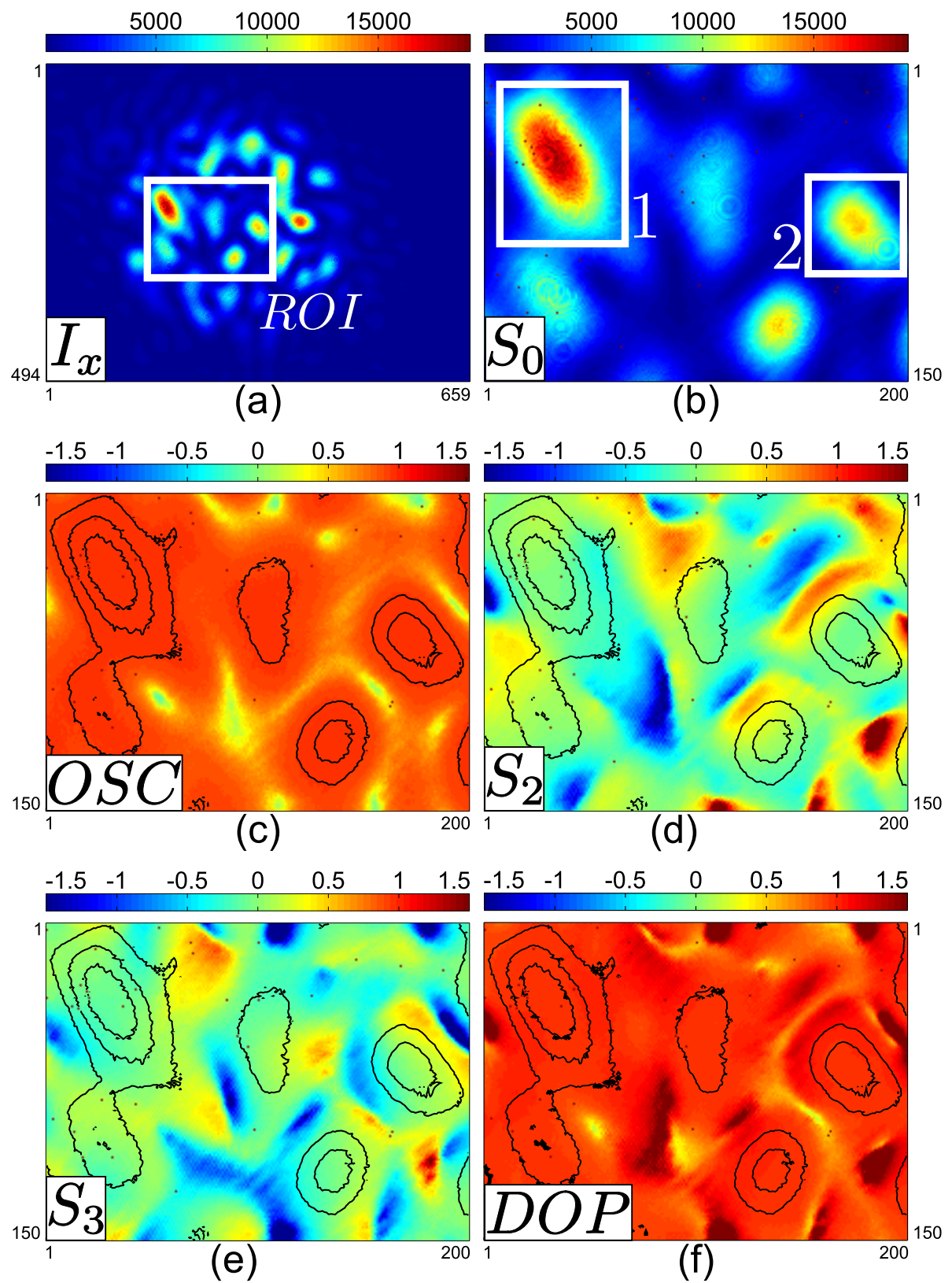

Fig. 9. Stokes imaging of a speckle intensity pattern obtained on the metallic reference slab. (a) : example of raw image acquisition $\left(I_{x}\right)$ and selection of the ROI. From the 6 acquisitions, the 4 Stokes images are determined: (b) First Stokes image $\mathcal{S}_{0}$ (total intensity). Two sub-ROIs are defined to compare the SOP in distinct speckle grains ; (c) Second normalized Stokes image $S_{1}$; (d) Third normalized Stokes image $\mathrm{S}_{2}$; (e) Fourth normalized Stokes image $\mathrm{S}_{3}$; (f) Degree of polarization. Total intensity repartition is indicated in contour plots in (c),(d),(e) and (f) thumbnails. 

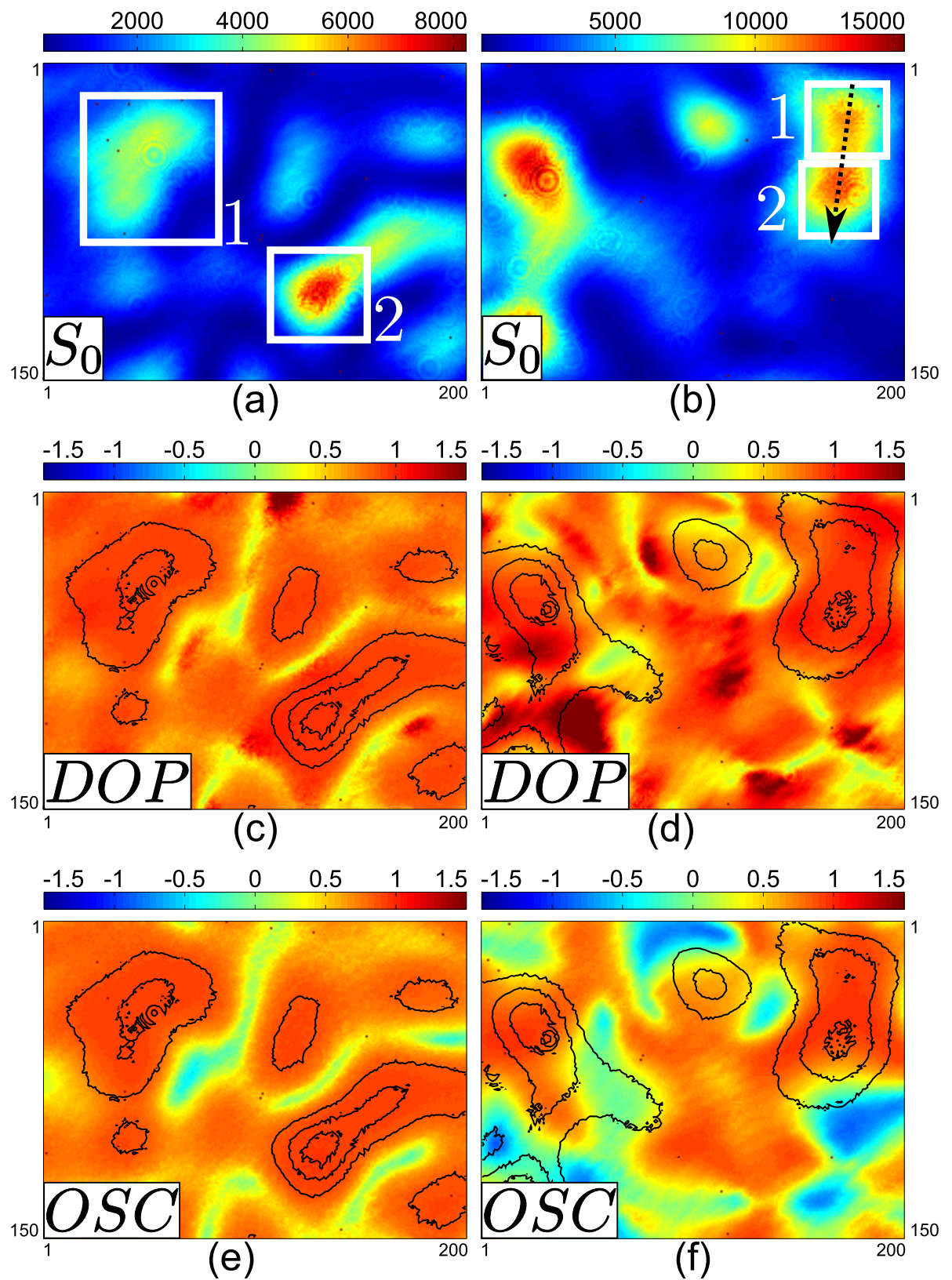

Fig. 10. Stokes imaging beyond the speckle grain scale of a speckle intensity pattern obtained on a red paint sample [(a), (c) and (e)] and a green paint sample [(b), (d) and (f)]. (a), (b): First Stokes image $\mathcal{S}_{0}$ (total intensity). Two sub-ROIs are defined to compare the SOP in distinct speckle grains ; (c), (d): Map of the computed DOP ; (e), (f): Map of the computed OSC. Total intensity repartition is indicated in contour plots in (c), (d), (e) and (f) thumbnails. 


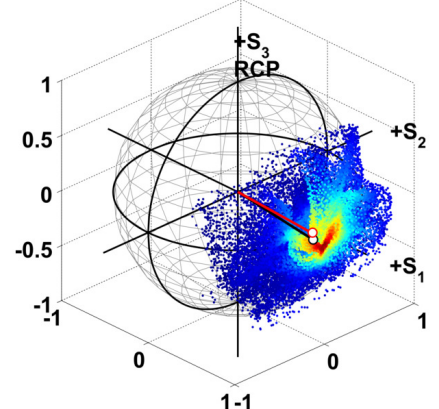

(a)

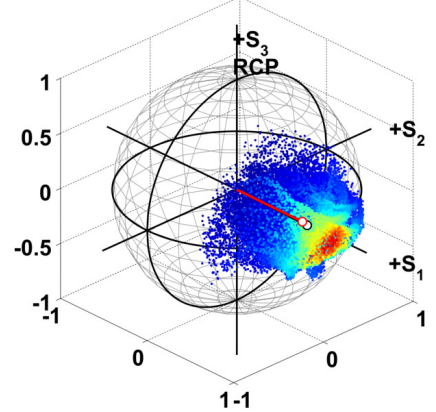

(d)

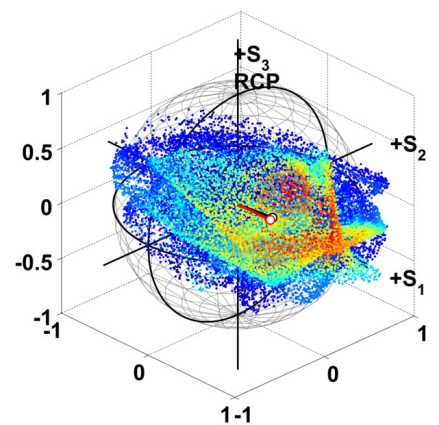

(g)

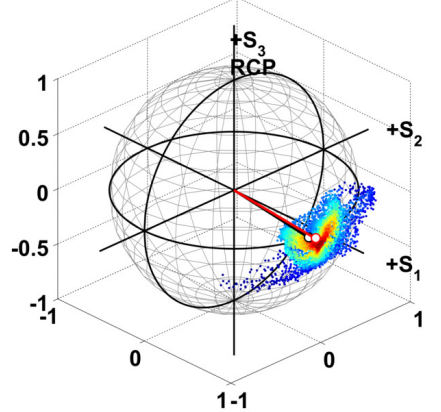

(b)

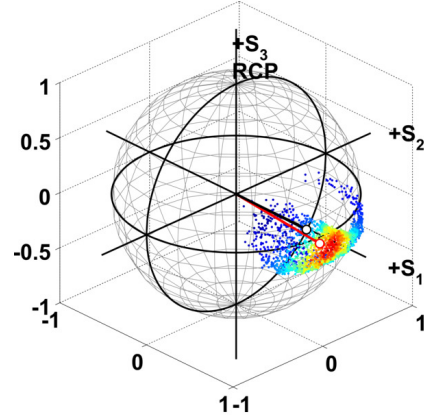

(e)

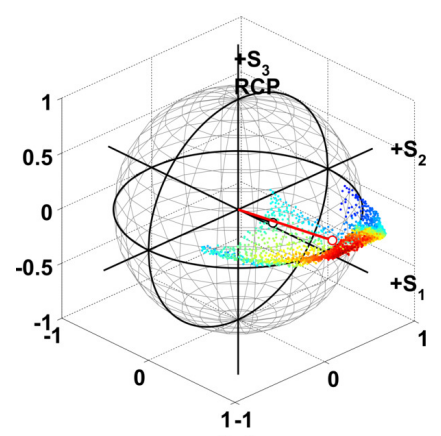

(h)

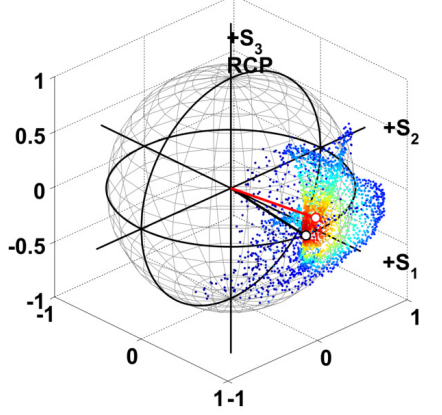

(c)

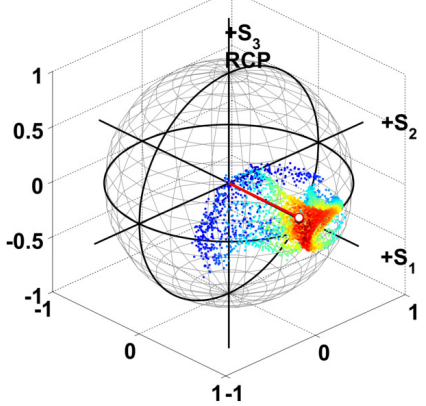

(f)

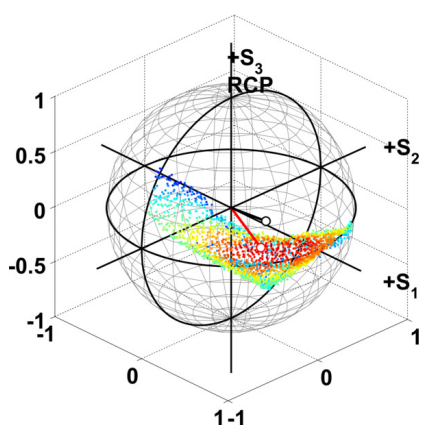

(i)

Fig. 11. Repartition of the pixels SOP on Poincaré's sphere for a metallic sample (first line), a red paint sample (second line) and a green paint sample (third line). First column: SOP in the whole ROI; Second column: SOP in sub-ROI 1 ; Third column: SOP in sub-ROI 2. Sub-ROIs are respectively defined in Fig. 9.b, Fig. 10.a and Fig. 10.b. 


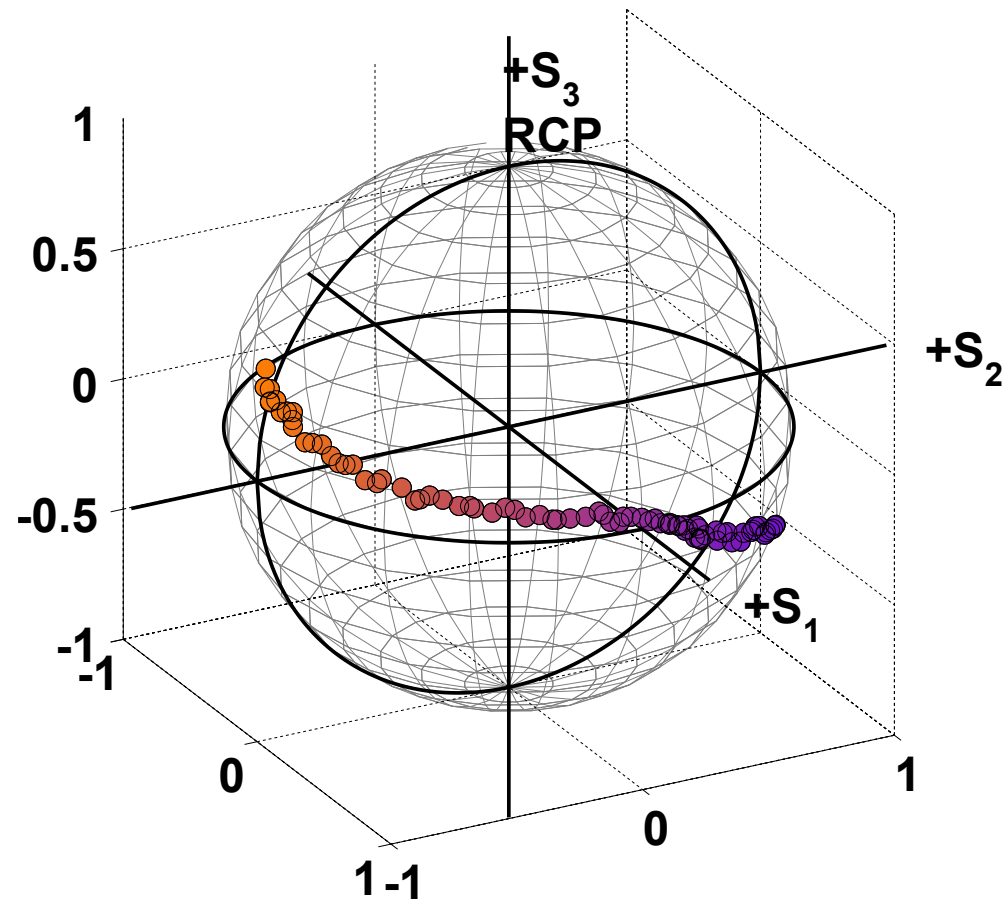

(a)

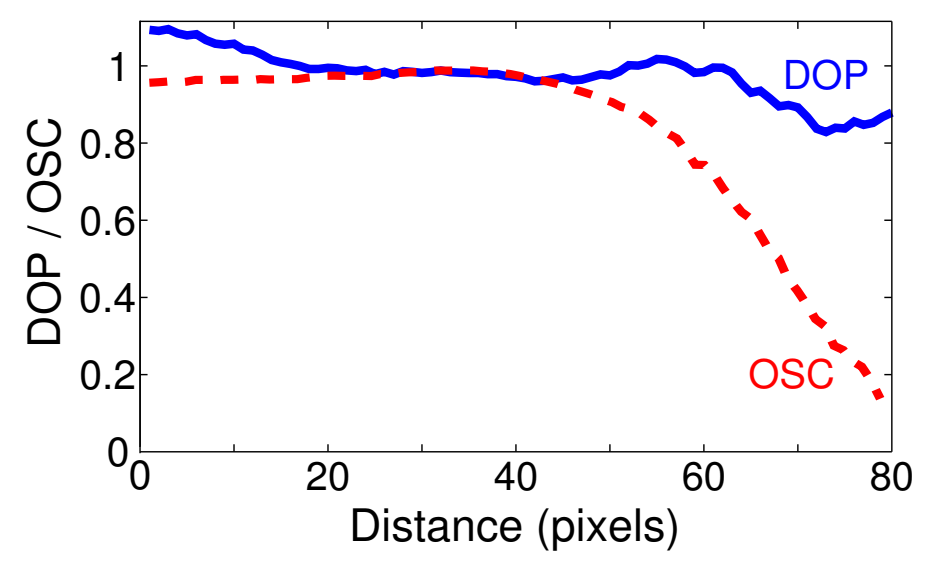

(b)

Fig. 12. Observation of the adiabatic polarization state transition along the geometrical path defined in Fig. 10.b between two adjacent speckle grains on the green paint sample exhibiting bulk scattering regime ; (a) Evolution of the SOP on the Poincaré's sphere; (b) Evolution of the OSC (dotted line) and DOP (plain line). 


\section{TABLE CAPTIONS}

Table 1: Large scale polarimetric characterization of light backscattered by the 3 samples considered. A maximum standard deviation of 0.02 was obtained over these 12 estimated values (Estimation on 420 homogeneous regions of 100 pixels).

Table 2: Average value of the DOP and of the OSC for different binning pitches. The estimated value and the corresponding standard-deviations were estimated in the whole ROI on a varying number of binned regions. 


\begin{tabular}{lcccc} 
Sample & DOP & $\mathrm{S}_{1}=\mathrm{OSC}$ & $\mathrm{S}_{2}$ & $\mathrm{~S}_{3}$ \\
\hline Metal slab & 0.97 & 0.96 & 0.12 & 0 \\
Red paint & 0.95 & 0.94 & 0.16 & -0.01 \\
Green paint & 0.44 & 0.43 & 0.07 & -0.01 \\
\hline
\end{tabular}

Table 1. Large scale polarimetric characterization of light backscattered by the 3 samples considered. A maximum standard deviation of 0.02 was obtained over these 12 estimated values (Estimation on 420 homogeneous regions of 100 pixels). 


\begin{tabular}{lcccccc}
\hline Sample & Bin. & Binned & \multicolumn{2}{c}{ DOP } & \multicolumn{2}{c}{ OSC } \\
& pitch & areas & $\langle\rangle$. & $\sigma$ & $\langle\rangle$. & $\sigma$ \\
\hline metal & 3 & 3333 & 1.00 & 0.23 & 0.85 & 0.17 \\
slab & 10 & 300 & 0.97 & 0.16 & 0.86 & 0.14 \\
& 30 & 33 & 0.93 & 0.06 & 0.91 & 0.07 \\
\hline red & 3 & 3333 & 0.80 & 0.18 & 0.69 & 0.23 \\
paint & 10 & 300 & 0.80 & 0.16 & 0.71 & 0.21 \\
& 30 & 33 & 0.80 & 0.10 & 0.77 & 0.12 \\
\hline green & 3 & 3333 & 0.78 & 0.30 & 0.38 & 0.47 \\
paint & 10 & 300 & 0.76 & 0.28 & 0.39 & 0.45 \\
& 30 & 33 & 0.61 & 0.24 & 0.41 & 0.36 \\
\hline
\end{tabular}

Table 2. Average value of the DOP and of the OSC for different binning pitches. The estimated value and the corresponding standard-deviations were estimated in the whole ROI on a varying number of binned regions. 